\title{
Dust formation as a diagnostic of Wolf-Rayet winds
}

\author{
Peredur M. Williams ${ }^{1}$, Karel A. van der Hucht ${ }^{2}$, Patrick W. Morris ${ }^{3}$, \\ and Fred Marang ${ }^{4}$ \\ ${ }^{1}$ Institute for Astronomy, University of Edinburgh, \\ Royal Observatory, Blackford Hill, Edinburgh EH93HJ, Scotland, UK \\ ${ }^{2}$ SRON Nationaal Instituut voor Ruimteonderzoek, \\ Sorbonnelaan 2, NL-3584 CA Utrecht, Nederland \\ ${ }^{3}$ SIRTF Science Center, IPAC, California Institute of Technology, \\ Pasadena, CA 91125, U.S.A. \\ ${ }^{4}$ South African Astronomical Observatory, \\ P.O. Box 9, Observatory, 7935, South Africa
}

\begin{abstract}
Dust formation by Wolf-Rayet stars is a powerful diagnostic of exceptional activity in their winds. Observed behaviour ranges from stars which make dust continually (e.g., WR 104, WC9d+B0.5V) to those which make dust for short episodes at periodic intervals (e.g., WR 140, WC7pd+O4-5). In both examples, dust formation is related to colliding WR and OB stellar winds in binary orbits. We have been using IR photometry primarily from the SAAO and ESO and spectroscopy with ISO-swS to investigate systems having intermediate properties (WR 98a, WC8-9vd+?) or more complex behaviour (WR.48a, WC8ed+?).
\end{abstract}

\section{Introduction}

Observations of infrared dust emission from Wolf-Rayet stars, and the recognition that the dust is formed in situ, have evolved from a curiosity to a problem to a diagnostic. The problem is the great difficulty of forming dust near such luminous stars: homogeneous stellar winds in the regions where grains are observed to exist are not dense enough to allow grains to form (Williams, van der Hucht \& Thé 1987; Cherchneff et al. 2000). Dust formation by WC stars requires that their winds are not homogenous, but contain regions of significantly higher density. The diagnostic comes from identifying these high-density regions and using the observations of dust emission to learn about the processes and stellar systems. Infrared photometric histories, some spanning more than two decades, show properties ranging from persistent dust formation at apparently constant rates, e.g., WR 104 (Ve 2-45, WC9d+B0.5V) to episodic dust formation occurring at regular intervals, e.g., WR 140 (HD 193793, WC7pd+O4-5) (Williams et al. 1987; Williams 1999). Dust formation in both these prototypes has been observationally linked to binary interactions. High-resolution $K$-band imaging of WR 104 by Tuthill, Monnier \& Danchi (1999) shows the dust to be formed in a rotating, collimated stream, forming a 'pinwheel', as from a low-inclination 

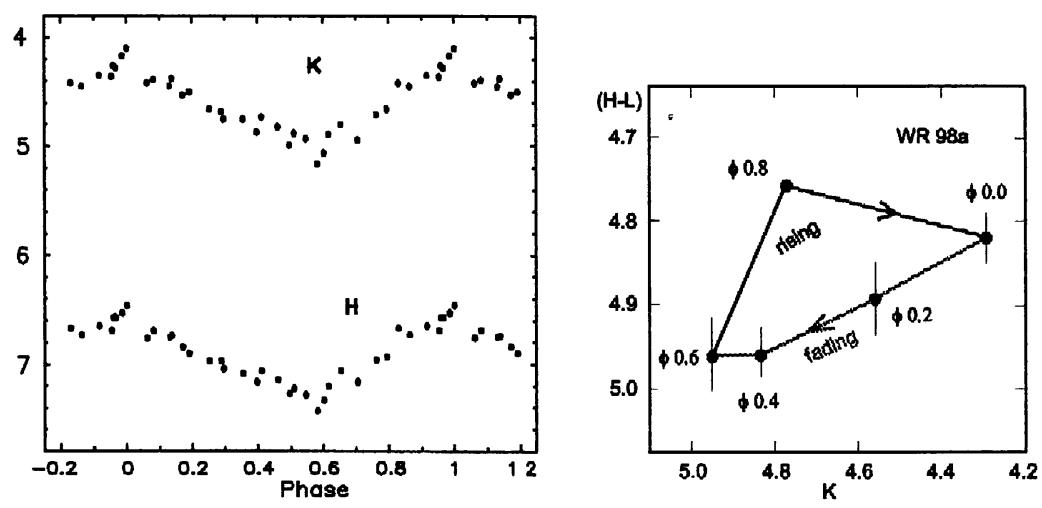

Figure 1. $H K L$ photometry of WR 98a (WC8-9vd+?) phased to a 564-day period and showing cycle in $(H-L)$ vs. $K$ (right) colour-magnitude field.

WC9+O binary. Long-term photometry of WR 140 shows periodic $(P=7.94 \mathrm{yr})$ episodes of dust formation occurring near periastron passage in the eccentric WC7+O4-5 binary orbit (Williams et al. 1990). High-density structures in these binary systems are provided by shocks where the winds collide (Usov 1991). The link between the dust formation episodes and binary orbit in WR 140 comes from the movement of colliding-wind region closer to the WC7 star (Williams 1999) during periastron passage. The apparently constant dust formation by WR 104 is consistent with the stars maintaining constant separation in a circular orbit.

Besides other persistent and episodic dust-making WC objects (WCd) resembling these prototypes (for an inventory of all known WCd stars, see the Galactic WR catalogue of van der Hucht 2001), there are systems showing more complex behaviour, such as WR 98a, another 'pinwheel' (Monnier, Tuthill \& Danchi 1999) but which shows variable dust formation. The infrared light curves of the episodic dust maker WR 137 (HD 192641, WC7pd+O9, $P=13.05 \mathrm{yr}$ ) also shows 'mini' dust-formation episodes which might be related to long-lived structures in a flattened wind (Williams et al. 2001) while those of WR 48a and WR 70 (HD 137603, WC9vd+B0I, see poster of Williams et al. in these Proceedings) are altogether more complex. Here we discuss new observations of WR 98a and WR 48a.

\section{WR 98a (IRAS 17380-3031, WC8-9vd+?)}

IRAS 17380-3031 was identified as a WR star (and listed WR 98a) by Cohen et al. (1991), and classified WC8-9. Infrared photometry from ESO and UKIRT in 1990-93 showed WR 98a to be variable (Williams et al. 1995) and it has been observed with the 0.75-m telescope at the SAAO since 1995. We did not attempt to combine the data on the different photometric systems because the colour equations ( $c f$. Carter 1990) are not defined for objects as red as WR 98a, and here consider the SAAO photometry on its own. These $J H K L$ data gave a period $P=564 \pm 8 \mathrm{~d}$, which is consistent with the earlier ESO-UKIRT data and is in excellent agreement with the 'pinwheel' rotation period $(P=565 \pm 50 \mathrm{~d})$ 


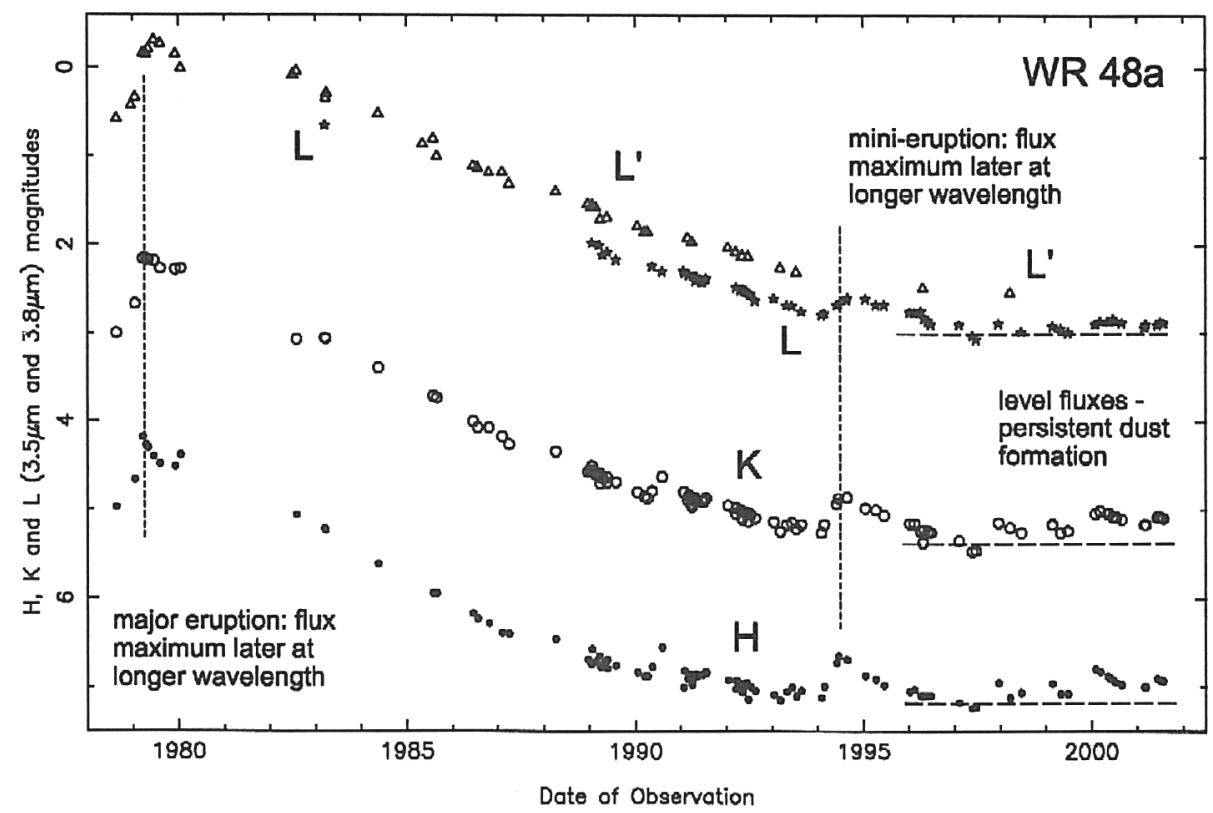

Figure 2. Synoptic infrared light curves of WR 48a (WC8ed+?) showing the major outburst in 1988-89 followed by slow fading to 'quiescent' levels characteristic of persistent dust formation. The fading is interupted by several 'mini' eruptions.

determined by Monnier et al. (1999). Phased $H K L$ light-curves and the track of WR 98a in an infrared colour-magnitude plane are shown in Figure 1. The dust temperature, as indicated by $(H-L)$, is significantly higher (at the same $K$ ) while the flux is rising than when it is fading, demonstrating that the infrared variation is caused by variation in the dust formation rate rather than orbital modulation of the extinction to the dust 'pinwheel' ( $c f$. Monnier et al. ). The fraction of WR stellar wind condensing into dust is a sensitive function of the separation of the two stars (Usov 1991), suggesting that WR 98a has an elliptical orbit. The light curves (Figure 1) are asymmetric because dust emission lags behind dust formation as the grains cool in the expanding wind.

\section{Dust-formation history of WR 48a (WC8ed+?)}

Long-term photometry (Figure 2) of WR 48a from ESO and the SAAO shows gradual fading from its major dust-formation episode in 1978-79, when it was discovered by Danks et al. (1983), to a 'quiescent' level about 3 mag fainter but still having a spectral energy distribution characteristic of continuing dust formation. This behaviour differs from that of episodic dust-makers like WR 140, which fade to stellar-wind spectra between eruptions. The fading was interupted by several 'mini' eruptions, secondary dust-formation episodes (e.g., 1994 - 95) having light-curve forms very similar to that of the 1978-79 major eruption: 


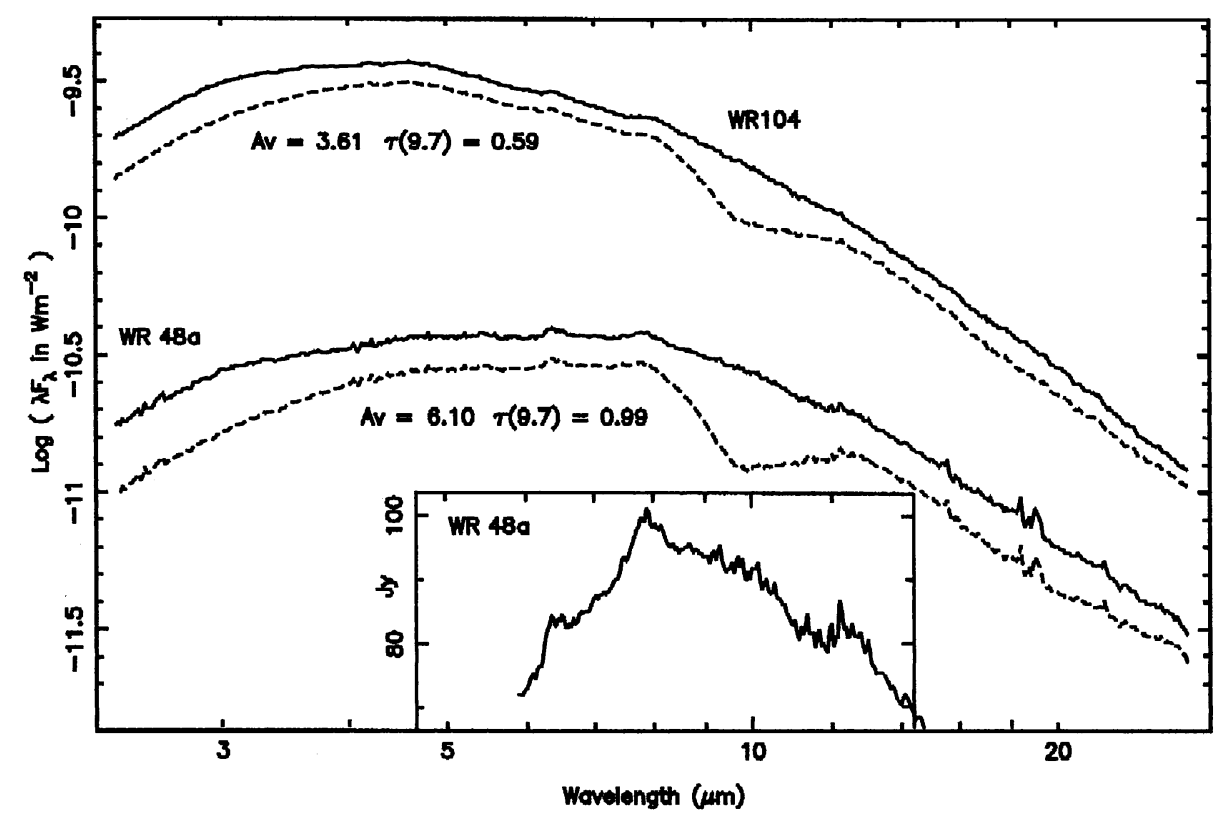

Figure 3. ISO-SWS spectra of WR $104(\mathrm{WC} 9 \mathrm{~d}+\mathrm{B} 0.5 \mathrm{~V})$ and WR 48a (WC8ed+?), as observed (dashed lines) and de-reddened (solid lines) by nulling the 'silicate' features and using the extinction law determined by Moneti et al. (2001). Inset: the de-reddened spectrum of WR 48a in linear flux units to show better the region of the 7.7- $\mu \mathrm{m}$ feature.

sharp rises to maxima occurring a little later at longer wavelengths, followed by slower declines. These 'mini' eruptions resemble those shown by the episodic dust-maker WR 137 (Williams et al. 2001), where they were ascribed to structures in the flattened wind of that star being crossed by the colliding wind zone during orbital motion. The rates of fading of the infrared emission from WR 48a are unusually slow: about $0.26 \mathrm{yr}^{-1}$, compared with $0.86 \mathrm{yr}^{-1}$ for WR 98a. The principal cause of the fading is the cooling of the dust as it is carried away by the wind and the stellar radiation heating it is further diluted. The rate of fading depends on the ratio of the stellar wind velocity to the scale of the dust cloud, specifically, the minimum distance from the stars at which the dust can exist in their radiation field, which depends on their luminosity. We do not know the wind velocity for WR $48 \mathrm{a}$, but it is unlikely to be much smaller than that of WR 98a $\left(\sim 900 \mathrm{~km} \mathrm{~s}^{-1}\right.$, Williams et al. 1995) given the average of $\sim 1150 \mathrm{~km} \mathrm{~s}^{-1}$ for the terminal velocities of WC9 stars (Eenens \& Williams 1994). The scale of the WR 48a dust cloud could be greater than that of WR 98a if the stellar luminosity heating it was greater, e.g., if the WC9 star in WR 48a had one or more companions more luminous than that of the WC8-9 star in WR 98a. The persistence of dust formation after fading from the 1978-79 outburst suggests the presence of a short-period $(P \simeq 1 \mathrm{yr})$ binary like those in WR 98a and WR 104, while the major outburst could have been caused by interaction with a third component in a longer period $(P>23 \mathrm{yr})$ orbit. 
Another possible cause for the slow fading of WR 48a is the continuation of dust formation at a declining rate after the 1978- 79 eruption. This would result in a rather different radial density distribution of the dust, assuming the cloud to be expanding at constant velocity, which maps on to the infrared spectral energy distribution via the radial grain temperature distribution.

\section{ISO-SWS spectroscopy of WR 48a and WR 104}

Five WCL stars with the densest dust shells, including WR 48a, WR 98a and WR 104, were observed with the short-Wavelength spectrometer (sWS) on ISO (van der Hucht et al. 1996). Dust emission dominates the spectra of the WCL stars, allowing study of interstellar and circumstellar features including those from grain precursors and also, from the overall spectral shapes, the run of dust density with temperature. The de-reddened sws spectrum of WR 48a, observed in 1996.10 when the near-IR flux level was close to 'quiescence' is significantly 'cooler' than that of WR 104 (Figure 3). If the dust made by the two stars has the same properties, including condensation temperature, this difference is consistent with a radial dust density distribution around WR 48a flatter than the $\propto r^{-2}$ law expected for a system making dust at a constant rate, like WR 104 .

The most interesting spectral feature is that near $7.7 \mu \mathrm{m}$, observed by Cohen, Tielens \& Bregman (1989) in KAO-FOGS and IRAS-LRS spectra of WR 104 and WR 112 (GL 2104, WC9d+OB?) and ascribed to a carbonaceous carrier with small aromatic domains, expected to be a precursor to carbon grains (Cherchneff \& Tielens 1994). This is conspicuous in the spectrum of WR 48a (Figure 3).

\section{References}

Carter, B.S. 1990, MNRAS 242, 1

Cherchneff, I., Tielens, A.G.G.M. 1994, in: K.A. van der Hucht \& P.M. Williams (eds.) Wolf-Rayet Stars: Binaries, Colliding Winds, Evolution, Proc. IAU Symp. No. 163 (Dordrecht: Kluwer), p. 346

Cherchneff, I., Le Teuff, Y.H., Williams, P.M., Tielens, A.G.G.M. 2000, A\&A 357, 572

Cohen, M., Tielens, A.G.G.M., Bregman, J.D. 1989, ApJ (Letters) 344, L13

Cohen, M., van der Hucht, K.A., Williams, P.M., Thé, P.S. 1991, ApJ 378, 302

Danks, A.C., Dennfeld, M., Wamsteker, W.M., Shaver, P.A. 1983, A\&A 118, 301

Eenens, P.R.J., \& Williams, P.M. 1994, MNRAS 269, 1082

van der Hucht, K.A., Morris, P.W., Williams, P.M., et al. 1996, A\&A (Letters) 315, L193

van der Hucht, K.A. 2001, New Astron. Reviews 45, 135

Moneti, A., Stolovy, S., Blommaert, J.A.D.L., et al. 2001, A\&A 366101

Monnier, J.D., Tuthill, P.G., Danchi, W.C. 1999, ApJ (Letters) 525, L97

Tuthill, P.G., Monnier, J.D., Danchi, W.C. 1999, Nature 398, 486

Usov, V.V. 1991, MNRAS , 252, 49

Williams, P.M. 1999, in: K.A. van der Hucht, G. Koenigsberger \& P.R.J. Eenens (eds), Wolf-Rayet Phenomena in Massive Stars and Starburst Galaxies, Proc. IAU Symp. No. 193 (San Francisco: ASP), p. 267

Williams, P.M., van der Hucht, K.A., Thé, P.S. 1987, A\&A 182, 91 
Williams, P.M., van der Hucht, K.A., et al. 1990, MNRAS , 243, 662

Williams, P.M., Cohen, M., van der Hucht, K.A., et al. 1995, MNRAS , 275, 889

Williams, P.M., Kidger, M.R., van der Hucht, K.A., et al. 2001, MNRAS 324, 156

\section{Discussion}

LEITHERER: Is the ice feature in the spectrum related to the WC winds?

WiLliams: No; it must be interstellar.

MoffaT: What is the current evidence on the fraction of WC9 stars that are binaries and form dust? If there are WC9 stars that do not form dust, then one will have to find another mechanism besides wind-wind collisions to account for WC9 stars.

WILlIAMS: The episodic dust makers are all confirmed binaries or have indirect evidence for binarity, such as spectroscopic companions. Searches for binarity amongst the persistent dust makers, whether direct imaging or by spectroscopy, has been less successful and it seems that the incidence of binaries amongst WC9 stars may be lower than other WR stars. Three WC9 stars have not been observed to have circumstellar dust - we don't know if they are binaries, but they do show spectroscopic differences from the dust-making WC9 stars.

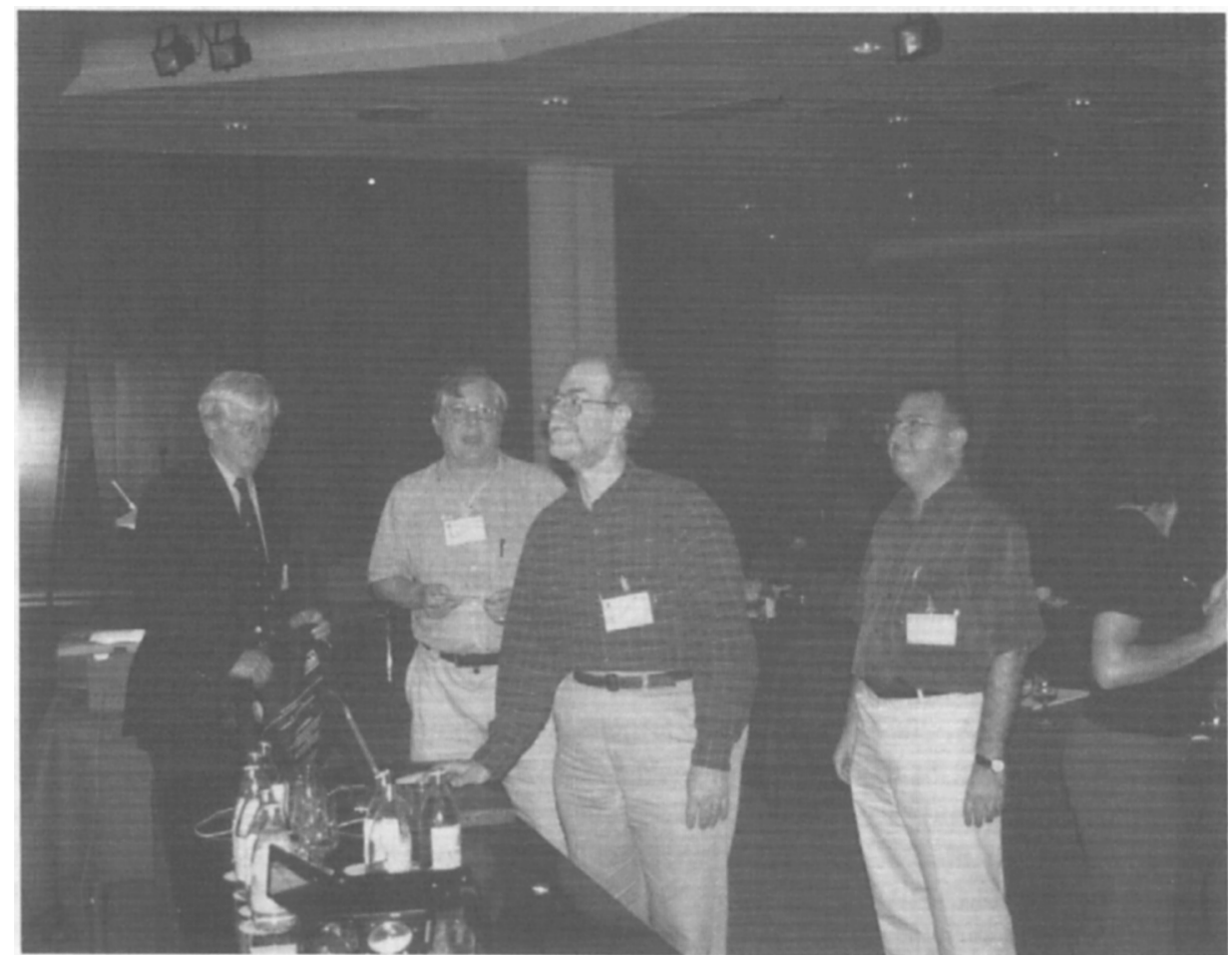

Are you using power-point or do you have something to say? Karel van der Hucht, Perry Williams, Phil Massey, Artemio Herrero and Charo Villamariz 